\title{
Design and validation of a colour food photography booklet for estimating food portion size in the traditional Mediterranean diet: contribution of traditional dishes to the Lebanese diet
}

\author{
M. Tueni ${ }^{1}$, A. Mounayar ${ }^{2}$ and I. Birlouez-Aragon ${ }^{3}$ \\ ${ }^{1}$ Polytechnic Institute LaSalle Beauvais, Beauvais, France, ${ }^{2}$ Lebanese University, Beyrouth, Lebanon \\ and ${ }^{3}$ AgroParisTech, Paris, France
}

\begin{abstract}
The aim was to design and validate a colour food photography booklet for estimating food portion size in Mediterranean countries and evaluating the contribution of traditional dishes in the Lebanese diet based on an FFQ.

The food photography booklet was constructed by taking digital photographs of three pre-weighed portions sizes of 212 traditional Lebanese dishes cooked according to traditional recipes. The booklet was validated by forty volunteers and 400 food portions were estimated. Each subject was asked by an interviewer to indicate the photograph best corresponding to the presented dish. The number of right answers or close to the right answer was counted to evaluate the precision of the portion size estimated. Using this tool, the daily intake of traditional dishes was quantified in a representative population sample of 566 Lebanese using an FFQ.

The majority of subjects (84\%) selected the correct or an adjacent portion as referring to the real portion size for the dish. For ten dishes eight series of photographs were regarded as satisfactory. The mean error acceptable at this level of estimation was $20 \%$. Linear regression analysis indicated that weights of portion sizes chosen from photographs were significantly associated $(P<0.05)$ with the real weights of the portions $\left(R^{2} 0.83\right)$. Bland-Altman plots showed good agreement between estimated and measured portions across all dishes, with a negligible bias of $0.73 \mathrm{~g}$. From the food-frequency study it was possible to estimate that $26 \%$ of the mean total daily energy intake was obtained from Lebanese traditional dishes. The contribution (\%) of traditional dishes to the total vegetable, legume and olive oil intake was calculated to be 43,98 and 70 respectively.

The validated food photography booklet is a useful tool to achieve acceptable quantification of consumption levels of traditional Mediterranean dishes. A validation of the nutritional composition will make it possible to derive the nutrient intake from the traditional diet. In Lebanon the significant contribution of traditional home-cooked dishes to the total energy intake indicates that this country still follows a traditional Mediterranean diet.
\end{abstract}

